\title{
Measurement Of Seam Puckering And Influence Of Its Causes
}

\author{
Sandun Fernando; Tss Jayawardena \\ Department of Textile and Clothing Technology, University of Moratuwa \\ Katubedda Road, 10400, Moratuwa, Sri Lanka.
}

\begin{abstract}
Seam quality is very important aspect of any form of textile assembly using sewing. The quality of the seam is governed by the seam puckering, which mainly depends on the technological parameters of the sewing machine and fabric specifications. Though this dependency is known in qualitative aspect, very little researches have been focused to investigate the quantitative aspect of seam puckering behavior of the seams. The Phong's shading algorithm based luminosity model was developed to estimate the dimensional parameters from the image of the seam captured and, an investigation has been carried out to analyze the dimensional characteristics of puckered fabric using image processing techniques and neural networks. This objective assessment attempt goes beyond the replacement of conventional subjective puckering evaluation methods of seams and move towards the development of empirical models for the causal effects quantitatively. That is the determination of correct needle thread tension setting which is the most influential machine parameter for seam puckering, for a minimum puckering.
\end{abstract}

Keywords: seam quality, puckering, image processing, technological parameters, dimensional parameters

\section{INTRODUCTION}

Garment related manufacturing industry is facing an era in which the quality, cost and compliance of the production are received a paramount importance. Apparently better quality and lower cost are the most basic factors for the success of this business wherever it is operated. Challenge is to reducing cost while maintaining the quality standards. Quality is given a main importance in the garment assembly. Sewing machine settings should be adjusted for a new style as the parameters such as fabric, sewing thread stitch density etc are different from the previous style to acquire better seam appearance. Machine set time between different styles of garments has been identified as a main point of wastage nowadays as style changes are frequent. Seam puckering is the most prominent aspect of seam which hinders the aesthetic appeal of the seam. Seam puckering is the effect of bulging of the fabric in the vicinity of the seam line. Seam puckering has unattractive appeal to textiles and found to be a severe problem in lightweight fabrics especially with low bending rigidity. Although elimination of seam pucker entirely not practical, high priority is given to control it within acceptable limits mainly using, sewing tension and stitch density.

The reduction of machine set times is hard to be achieved as there is no proper method to identify the right machine settings depending on the fabric and other technological parameters to achieve acceptable degree of seam puckering straightaway. Because of this reason, it's required to depend on the trial and error method extending the machine set times leading to a huge wastage of time, expensive fabrics, threads, electricity and all. In a competitive environment the designer anticipated aesthetic appeal must be acquired in a minimum expenditure of time and resources.

The main reason for seam puckering was identified as the use of higher tension settings in sewing. The over stretched thread in the stitch tend to relax to get its original length leaving a bulging effect and structural jamming of fabric between the thread insertion points. Many other operational parameters and physical parameters of material used for sewing have a great influence on seam puckering and most dominant technological parameters responsible for puckering were under investigation.

In this paper, an objective seam puckering assessment method is proposed with image processing techniques to overcome the subjectiveness of assessment and further investigates the influence of several important technological parameters of sewing such as the needle tension variation and thread/fabric type for seam puckering using a neural network model. No relationship was found in literature about the intensity of seam puckering and its root causes quantitatively. The scope of the research was limited to fabric types of plain woven $100 \%$ Cotton fabrics and to standard lockstitch machine.

F.N Ferreira, S.C Harlock and P. Grpsberg, in 1994 has used thread tension sensors and some other auxiliary hardware to measure the tension of both bobbin and needle thread [01],[02]. He was successful in identifying the the tension behavior of the needle and bobbin thread within a stitch cycle and noticed that there are several peaks in the profile. A similar project but with rather different approach was done by T. Horino, Y.Miura, Y. Ando, K. Sakamoto, in year 1982[03] by Simultaneous measurements of needle thread tension and check spring motion of lockstitch sewing machine. Several other similar projects were found in literature 
but still needs lot of sophistications for the practical usability. In the puckering analysis, several interesting projects were carried out, specially in the areas of neural networks [04], fabric simulation and structural physics of textile fabrics. We intend to objectively analysis the seam puckering and thus move towards the root causes of it quantitatively with a neural network.

An attempt was made to investigate the effect of fabric and sewing conditions on apparel seam characteristics [7]. Sewing speed, stitch length, sewing thread tension, and presser foot pressure were selected as sewing parameters attempted optimizing sewing conditions to minimize seam pucker using the Taguchi method. [8]. Further, Choudhary et. al. Investigated the effect of fabrics and sewing conditions on apparal seamcharacteristics [12]. The influence of mechanical properties of yarn such as tensile strength on seam pucker of lightweight fabric was evaluated after washing and drying with comparison [11]. Experimental comparison of seam performance in different polyester and cotton woven fabrics was made using two types of sewing threads [9]. A statistical approach was carried out to determine the effects of the selected sewing parameters in denim fabric on seam strength. However, it was not investigate the puckering effect as denim which is less prone to seam puckering [10].

\section{METHODOLOGY}

The main technological parameters that were tested in the research were pulley tension setting, needle thread tension and stitch density. Because the needle thread tension depends on the bobbin thread tension, bobbin thread tension was kept at a constant at a standard tension value in order to isolate. The main fabric specifications tested were, fabric GSM, bending rigidity of the fabric, and Young's Modulus of the fabric. To obtain a good variation range of these fabric parameters, seven plain woven fabrics were selected with different parameter values. Six fabrics were used to observe the behavior of the seam characteristics with machine tension related parameters and one fabric is tested with changing stitch density. For each sewing condition of the fabric, six to ten replicates were produced with the approximate dimensions of $11 x 1.5$ inches. All the fabric samples were sewn in the warp direction, using standard sewing thread counts, needle sizes, under constant bobbin thread tension. The specifications such as material, Tex count of the yarn, construction, and the weight of the seven fabric types selected were given in Table 1 .

Table 1: Specifications of different fabrics used

\begin{tabular}{|l|l|l|l|l|}
\hline & Material & Yarn (Tex) & Construction & weight $\left(\mathrm{g} / \mathrm{m}^{2}\right)$ \\
\hline Fabric 1 & $100 \%$ Cotton & $\begin{array}{l}\text { Weft -20 Tex } \\
\text { warp -20 Tex }\end{array}$ & $\frac{20 \times 20}{100 \times 52}$ & 200 \\
\hline Fabric 2 & $100 \%$ Cotton & $\begin{array}{l}\text { Weft- 20 Tex } \\
\text { warp -20 Tex }\end{array}$ & $\frac{125 \times 64}{20 \times 20}$ & 279 \\
\hline Fabric 3 & $100 \%$ Cotton & $\begin{array}{l}\text { Weft -30 Tex } \\
\text { warp -30 Tex }\end{array}$ & $\frac{68 \times 68}{30 \times 30}$ & 116 \\
\hline Fabric 4 & $100 \%$ Cotton & $\begin{array}{l}\text { Weft -30 Tex } \\
\text { warp -30 Tex }\end{array}$ & $\frac{148 \times 80}{30 \times 30}$ & 214 \\
\hline Fabric 5 & $100 \%$ Cotton & $\begin{array}{l}\text { Weft -40 Tex } \\
\text { warp -40 Tex }\end{array}$ & $\frac{133 \times 72}{40 \times 40}$ & 138 \\
\hline Fabric 6 & $100 \%$ Cotton & $\begin{array}{l}\text { Weft -40 Tex } \\
\text { warp -40 Tex }\end{array}$ & $\frac{130 \times 100}{40 \times 40}$ & 160 \\
\hline Fabric 7 & $100 \%$ Cotton & $\begin{array}{l}\text { Weft -40 Tex } \\
\text { warp -30Tex }\end{array}$ & $\frac{120 \times 100}{40 \times 30}$ & 172 \\
\hline
\end{tabular}

\subsection{Experimental conditions and setup}

For the experiment $100 \%$ cotton plain woven fabrics were selected as they cover standard fabric weight ranges. The polyester thread was selected as the needle as well as bobbin thread throughout the experiment. The standard sewing conditions used were summarized in Table 2. 
Table 2: Sewing conditions for the experiment

\begin{tabular}{|l|l|}
\hline Stitch type & 301 lockstitch \\
\hline Speed & $1,500 \mathrm{rpm}$ \\
\hline Bobbin thread tension & $75 \mathrm{gf}$ \\
\hline Needle sizes & $65,70,100$ \\
\hline Sewing needle count & 24,40 \\
\hline Feed rate & $7-8 \mathrm{~cm} / \mathrm{s}$ \\
\hline
\end{tabular}

The bobbin thread tension was set initially before carrying out the experiments with a specially designed frame using dead weights for calibration. Another frame was developed to accommodate electronic tension measuring instrument of strain gauge type. It gives a response rate of 90-100 Hz. All the samples were kept in conditioned environment with $25^{\circ} \mathrm{C}$ temperature and 55\% RH. All the samples are pressed and left for relaxation over one day period prior to assessment of seam puckering.

Three major variations were tested in this research. First fabric samples of different weights were tested under varying the tension pulley setting in the sewing machine while keeping all the other parameters constant. Secondly, the fabric quality is selected to test the puckering behavior of the fabric with varying stitch density while keeping all the other parameters constant. Thirdly, an experiment was done to observe the needle thread tension profile for a selected fabric as stitch density varied.

Images of these samples were captured with an optical scanner with 300 dpi resolution in both directions and in 8-bit grayscale. The dimensional behavior of the seam against the varying operational and fabric parameters were observed by analyzing the captured images with image processing techniques and objectively grade the intensity of puckering.

\subsection{Analysis of dimensional properties of puckered fabric}

Scanned images produced by the experiments are used to analyze the dimensional properties of the actual puckered fabric. In the fabric puckering standard developed by AATCC [05] (American associations of Textile Chemist and Colorists) has five images with ascending order of the severity of seam pucker. User needs to compare the samples to be evaluated against the standard image with the eye and select the best matching image. Rating of puckered sample is the rating of matched standard image. For such evaluation system, the dimensional characteristics of the actual deformed fabric have not been taken into account in addition to its subjectivity.

Considering above limitations an objective evaluation method was developed and it can describe the seam puckering based on the dimensional characteristics. Seam puckering in a fabric can be identified as wave front which originates from the seam-line and propagates some distance along the fabric. The captured image of the seam is processed with image processing operations such as contrast adjustment, adaptive thresholding and some morphological operations to isolate the tone variation from random effects and noises. The variation of the tone in the processed image, along a line parallel to the seam line is used to describe the dimensional characteristic such as amplitude and wavelength of the puckering wave. Several such lines were considered in different distances from the seam line and the variation of the puckered wave amplitude and the wavelength is estimated. The amplitude and wavelength variations parallel to the seam line (i.e. dimensional properties of the puckered fabric) characterize the severity of puckering and it can be used to determine the rating. The Phong's shading algorithm [06] was used in developing a luminosity model to estimate the dimensional parameters from the image. The model developed can be stated as

$$
y=a \sin (b x+c)+d
$$

Where $\mathrm{y}$ is the tonal amplitude in gray scale, $\mathrm{x}$ is the pixel distance along a parallel line to the seam from one end of the sample. a, b, c, d are estimated parameters of the luminosity model which represent amplitude, scaling parameter of frequency, phase shift and the offset value of the tonal amplitude respectively.

\section{RESULTS AND DISCUSSION}

\subsection{Estimation of luminosity model parameters}

The pixel intensity measured in gray scale in the image parallel to the seam line varies approximately in a sinusoidal wave pattern contaminated with noise. Figure 1 shows the raw gray scale intensity variation of pixels parallel to the seam line. Smoothened wave in Fig 2 could be obtained by taking the moving average of adjacent 
five pixel gray values of image data in Fig 1. Thus the noise could be removed and smoothened wave pattern so that it could be used to estimate the parameters of the luminosity model.

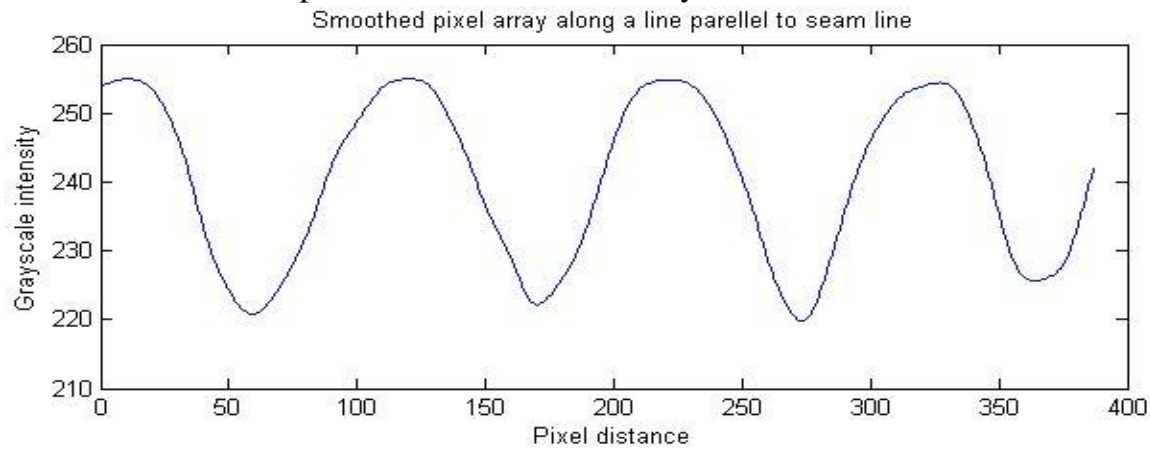

Figure 1: Raw pixel gray scale intensity variation

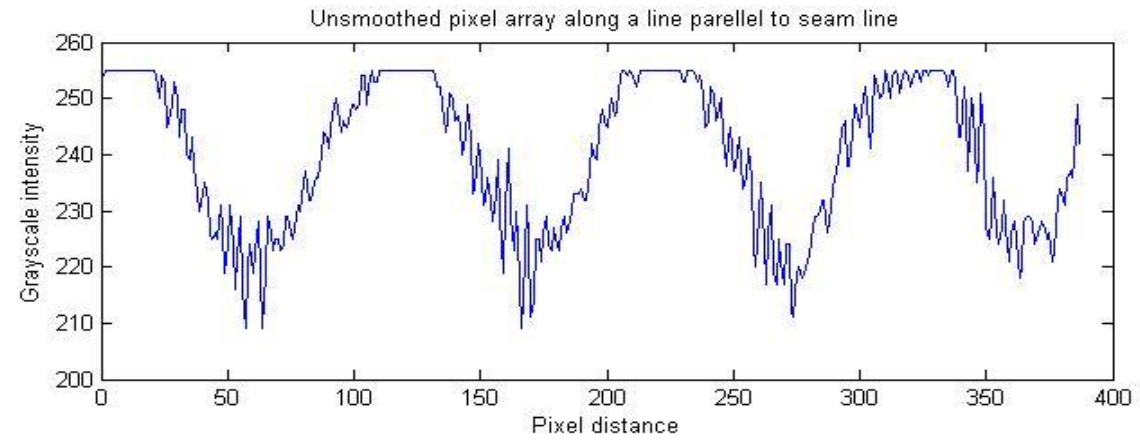

Figure 2: Smoothened pixel gray scale intensity variation

The Phong's shading algorithm based luminosity model described in equation 1 has four parameters a, $\mathrm{b}, \mathrm{c}$ and $\mathrm{d}$. The smoothened gray scale intensity variations were used to estimate the model parameters which represents the dimensional parameters of the seam described above. Take the average difference between the lowest and highest values of gray scale intensity and divide it by two to estimate the parameter ' $a$ '. The average of total pixel gray scale intensity values between two peak points could estimate parameter-' $\mathrm{d}$ '. The parameter ' $b$ ' was calculated by dividing the two times pi value by the pixel distance between two consecutive peaks of intensity variation. Having estimated parameters $\mathrm{a}, \mathrm{b}$ and $\mathrm{d}$, it is possible to estimate the parameter ' $\mathrm{c}$ ' for a particular point by fitting that point to the model. In this way, the parameter ' $\mathrm{c}$ ' for a set of points were estimated and get the average value to estimate the parameter ' $c$ ' of the model.

The goodness of fit of luminosity model developed was demonstrated graphically in Fig. 3 and statistically by obtaining the $\mathrm{R}$ squared value for the fitness of model. The sub arrays with similar lengths have provided relatively better fits with higher $\mathrm{R}$ squared values such as 0.75 or 0.8 and it was consistent over many other samples tested.

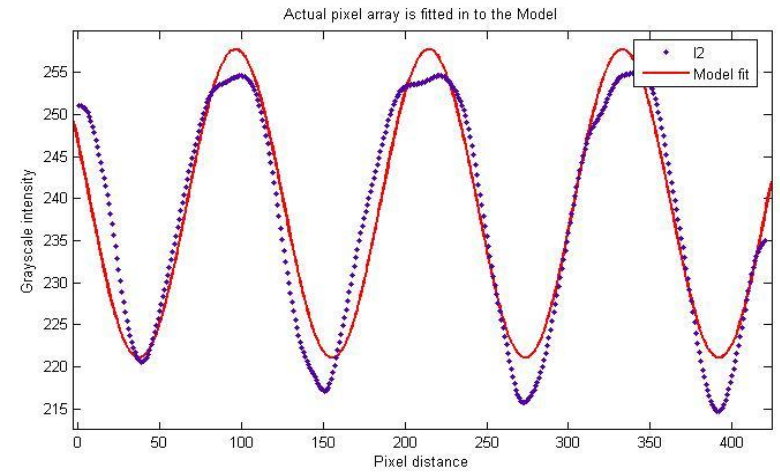

Figure 3: Comparison of actual tonal variation and predicted tonal variation using the model 


\subsection{Estimation of luminosity model parameters}

The key technological parameters, namely needle thread tension variation, GSM of the fabric and bending rigidity of fabric were tested and how they affect for the severity of seam puckering based on AATCC standards. The actual images were analyzed with an objective seam puckering grading system and results were organized in to respective groups and fitted it to a linear model using least square method.

The liner data model used for fitting can be given as

$y=P_{1} x+P_{2}$

where ' $y$ ' is the response variable of the fitted model and $x$ is the independent variable. P1 and P2 are the gradient and the intercept of the fitted model respectively. Two confidence intervals for P1 (P1 CI) and P2 (P2 CI) are calculated at 95\% confidence level. The coefficient of determination (R2) and adjusted coefficient of determination (Adjusted R2) were estimated to compare the goodness of fit of experimental data in to the fitted model.

In the case of fabric specifications, two main parameters were varied, they are fabric GSM and bending rigidity. The only machine technological parameter tested, the thread tension is provided by a spring and according to the hooks law, tension is directly proportional to the compression which can be represented by the number of rotations of the tension dial. The incremental tension setting of the machine above $75 \mathrm{gf}$ is provided in number of main tension dial rotations. Figure 4 shows the variation of AATCC grading with the machine tension setting and Table 3 shows the goodness of fitting.

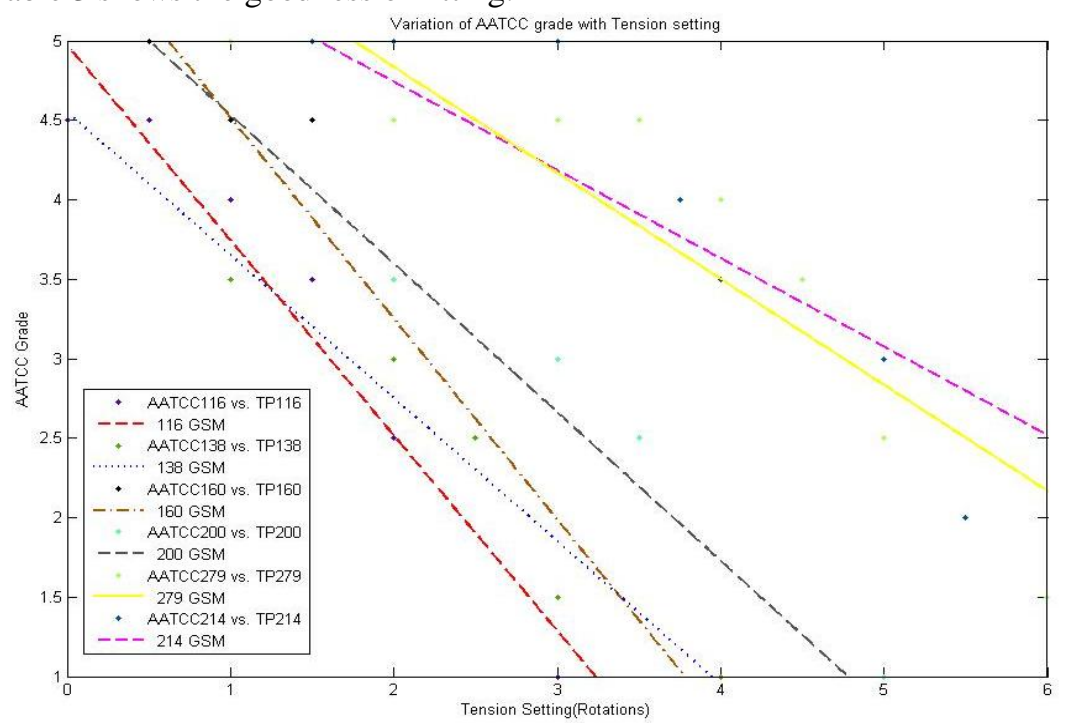

Figure 4: AATCC grading vs the machine tension

Table 3: Parameters for best fit model for AATCC grading vs machine tension and goodness of fit

\begin{tabular}{|c|c|c|c|c|c|c|}
\hline GSM & P1 & P2 & P1 $(\mathrm{Cl})$ & P2 (CI) & R2 & AdjustedR2 \\
\hline 116 & -1.23 & 4.97 & $(-1.646,-0.8107)$ & $(4.278,5.664)$ & 0.94 & 0.93 \\
\hline 138 & -0.9 & 4.55 & $(-1.311,-0.4891)$ & $(3.444,5.656)$ & 0.94 & 0.92 \\
\hline 160 & -1.27 & 5.78 & $(-1.685,-0.8443)$ & $(4.801,6.758)$ & 0.95 & 0.93 \\
\hline 200 & -0.93 & 5.47 & $(-1.321,-0.5486)$ & $(4.173,6.758)$ & 0.92 & 0.9 \\
\hline 214 & -0.56 & 5.85 & $(-0.7954,-0.3159)$ & $(5.043,6.663)$ & 0.78 & 0.75 \\
\hline 279 & -0.67 & 6.17 & $(-0.9822,-0.3511)$ & $(4.927,7.406)$ & 0.82 & 0.79 \\
\hline
\end{tabular}

Similarly the effect of fabric GSM for AATCC grading was tested under constant tension of the needle tread and the results are given in Fig.5. Table 4 represents the linear model fitting parameters of AATCC grading vs fabric GSM of the fabric. 


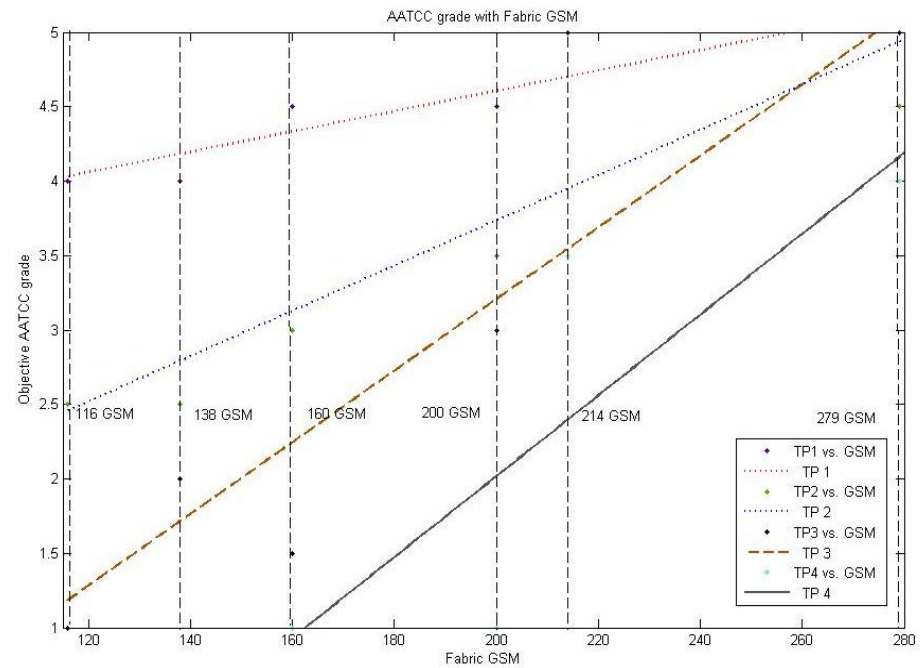

Figure 5: AATCC grading vs fabric GSM under constant tension setting

\begin{tabular}{|c|c|c|c|c|c|c|}
\hline Tension & $\mathrm{P} 1$ & $\mathrm{P} 2$ & $\mathrm{P} 1(\mathrm{Cl})$ & $\mathrm{P} 2(\mathrm{Cl})$ & $\mathrm{R} 2$ & Adjusted R2 \\
\hline 1 & 0.00766 & 3.76400 & $(0.004666,0.01065)$ & $(3.551,3.977)$ & 0.9838 & 0.9757 \\
\hline 2 & 0.01105 & 2.17400 & $(-0.008641,0.03074)$ & $(0.771,3.577)$ & 0.7445 & 0.6168 \\
\hline 3 & 0.01125 & 1.16100 & $(-0.04923,0.07172)$ & $(-3.148,5.47)$ & 0.2425 & -0.1362 \\
\hline 4 & $\mathrm{~N} / \mathrm{A}$ & 1.00000 & $\mathrm{~N} / \mathrm{A}$ & $(1,1)$ & 1.0000 & 1.0000 \\
\hline
\end{tabular}

With higher tension it was observed that puckering wavelength reduces. But this effect seems to be happening in different phases for fabrics with different GSM values. Figure 6 shows the variation of the needle thread tension on the wavelength of puckering and Table 5 gives the fitness of its modeling.

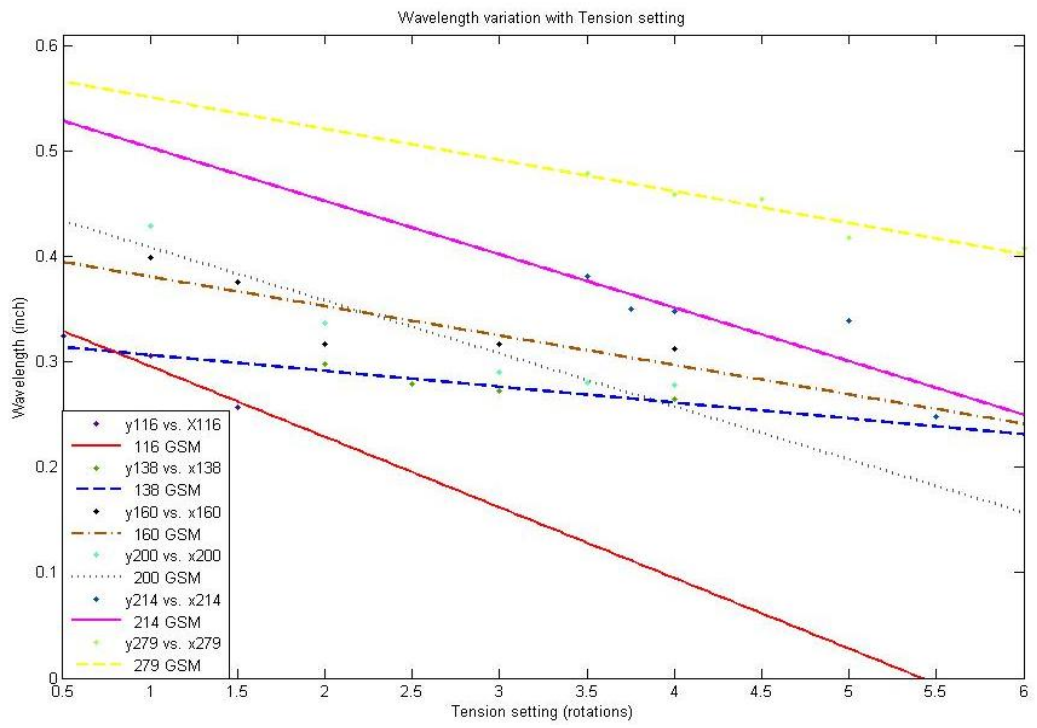

T\& Figure 6: Wavelength vs tension setting graph $\quad \mathrm{ng}$ 


\begin{tabular}{|c|c|c|c|c|c|c|}
\hline GSM & P1 & P2 & P1 (Cl) & P2 (Cl) & R2 & AdjustedR2 \\
\hline 116.000 & -0.07 & 0.36 & $(-0.2929,0.159)$ & $(0.1185,0.6066)$ & 0.9341 & 0.8681 \\
\hline 138.000 & -0.02 & 0.32 & $(-0.02282,-0.007277)$ & $(0.3003,0.3422)$ & 0.9268 & 0.9024 \\
\hline 160.000 & -0.03 & 0.41 & $(-0.06232,0.006586)$ & $(0.3207,0.4957)$ & 0.6883 & 0.5845 \\
\hline 200.000 & -0.05 & 0.46 & $(-0.08067,-0.0199)$ & $(0.3701,0.5468)$ & 0.9024 & 0.8698 \\
\hline 214.000 & -0.05 & 0.55 & $(-0.1038,0.002467)$ & $(0.3188,0.7881)$ & 0.7543 & 0.6724 \\
\hline 279.000 & -0.03 & 0.58 & $(-0.04586,-0.01361)$ & $(0.5046,0.6556)$ & 0.9198 & 0.8931 \\
\hline
\end{tabular}

\section{CONCLUSION}

In this research an objective measurement of seam puckering was done with image processing techniques. It is not just a replacement for subjective evaluation scheme, but a guide to adjust machine parameter- the needle thread tension as GSM and bending rigidity of the fabric varies in order to minimise seam puckering. In addition to image processing techniques a luminosity model was developed based on Phong's shading algorithm and the parameters of the model were estimated to extract the dimensional characteristics of the puckered seam. The cause and effect relationship was modeled empherically and statistical evaluation techniques were used to justify the fitness of the model. The results of the objective evaluation method developed enables us to determine the intensity of puckering and assess its corresponding grading. A guide can be developed to determine the best suited thread tension setting for minimum puckering conditions under varying GSM and the bending rigidity of the fabric based on the empherical models developed.

The research work presented in this paper has several limitations in its application. This work was tested only for white fabric seams only and it is required to extended for other fabrics with different mixture of colours. Use of IR sensor and IR sources may be helpful in such extension as reflection of IR light is not very much sensitive to the colour change of the fabric. This was tested only for standard lock stich machine and the validity of the method proposed is yet to be tested against the change in machine/stich type. The robustness of the proposed method should be assesses against the variation of the fabric type as well.

\section{REFERENCES}

[1] Ferreira, F. N., Harlock, S. C., Grosberg, P., A Study of Thread Tensions on a Lockstitch Sewing Machine (Part I), International Journal of Clothing Science and Technology, Vol. 6, No. 1, pp. 14-19, MCB University Press, U.K, 1994.

[2] Ferreira, F. N., Harlock, S. C., Grosberg, P., A Study of Thread Tensions on a Lockstitch Sewing Machine (Part II), International Journal of Clothing Science and Technology, Vol. 6, No. 1, pp. 14-19, MCB University Press, U.K, 1994.

[3] Horino, T., Miura, Y., Ando, Y., Sakamoto, K., Simultaneous measurements of needle thread tension and check spring motion of lockstitch sewing machine for industrial use, J. Text. Mach. Soc. Japan, vol. 2, pp. T30-37, 1982.

[4] K.L. MAK, WEI LI, Objective Evaluation of Seam Pucker on Textiles by Using Self-Organizing Map, IAENG International Journal of Computer Science, 35:1, IJCS_35_1_07.

[5] AATCC Test Methods 88B-1992, vol. 69, pp. 115.

[6] B. T. Phong, Illumination for computer generated pictures, Communications of ACM 18 (1975), no. 6, 311-317.

[7] Chang Kyu Park and Tae Jin Kang, Objective Evaluation of Seam Pucker Using Artificial Intelligence Part III: Using the Objective Evaluation Method to Analyze the Effects of Sewing Parameters on Seam Pucker, Textile Research Journal, December 1999 Volume 69, pp. 919-924

[8] Chang Kyu Park and Joo Yong Ha, A Process for Optimizing Sewing Conditions to Minimize Seam Pucker Using the Taguchi Method, Textile Research Journal, March 2005 Volume 75, pp.245-252

[9] Vildan Sülara, Cansu Meşegülb, Hülya Kefsizc and Yasemin Sekia, A comparative study on seam performance of cotton and polyester woven fabrics, The journal of the Textile Institute, March 2014, pp. $1-12$

[10] Belk1s Zervent Ünal, The prediction of seam strength of denim fabrics with mathematical equations, the journal of the Textile Institute, Volume 103, Issue 7, 2012, pp 744-751

[11] Vaida Dobilaite and Milda Juciene, The influence of mechanical properties of sewing threads on seam pucker, International Journal of Clothing Science and Technology, Vol. 18, No. 5, 2006, pp. 335-345

[12] A. K. Choudhary and Amit Goel Effect of Some Fabric and Sewing Conditions on Apparel Seam Characteristics,Journal of Textiles, Volume 2013 (2013), Article ID 157034. 